\title{
A possible extratubular origin of epididymal basal cells in mice
}

\author{
C. Holschbach and T. G. Cooper* \\ Institute of Reproductive Medicine of the University, Domagkstrasse 11, \\ D-48129 Münster, Germany
}

The origin of basal cells in mouse epididymis was examined by counting the numbers of basal cells, intratubular mitotic figures and peritubular cells during development of the epididymis. Putative precursors of basal cells were labelled with bromodeoxyuridine and the nuclei of daughter cells were examined. Histochemical localization of cytokines was performed to gauge their involvement in migration of basal cell precursors from extratubular sources. The results indicate that basal cells may arise from extratubular sources as: (i) there was a decrease in the number of mitotic figures as the number of basal cells increased; (ii) no mitotic figures were observed in the base of the epithelium; (iii) the increase in the number of peritubular cells did not parallel the number of basal cells in all epididymal regions; (iv) division of epithelial cells into daughter cells was circumferential and not radial; (v) bromodeoxyuridine-

\section{Introduction}

The epididymis appears to have a functioning secretory epithelium in early neonatal life, as the tubule has a lumen at a time when the testis contains only seminiferous cords. Further differentiation occurs before puberty, when the basal cells appear in the epithelium, and at puberty, when the initial segment differentiates (for references, see Seiler et al., 1998). In mice, there is 'ascending' maturation, meaning that basal cells appear in the cauda before the corpus epididymidis and in the corpus before the caput epididymidis (Seiler et al., 1998, 1999).

Although Martan (1969) proposed that basal cells are the progenitors of principal cells, the holocrine secretory cycle of which they were an integral part is now discredited and the time-course of their appearance, after that of principal cells, clearly excludes this possibility. The opposite view, that columnar (principal) cells give rise to basal cells, has been proposed by Sun and Flickinger (1982) in a study in which the time-course of appearance of all the types of cell in rat epididymis was monitored. However, the low mitotic activity in the epididymis (Clermont and Flannery, 1970; Sun and Flickinger, 1982; Sujarit and Jones, 1991) may not be compatible with such a view. An alternative explanation,

*Correspondence

Email: cooper@uni-muenster.de labelled basal cell nuclei were mostly not found in the vicinity of labelled principal cell nuclei and vice versa; and (vi) the percentage of labelled basal cell nuclei was higher than that of the other cells, which is indicative of their arrival from a more highly labelled pool. In addition, no age-dependent correlation was noted between any of the cytokines tested and appearance of basal cells in the epithelium, and basal cells expressed intercellular adhesion molecule 1 (ICAM-1) and vascular cell adhesion molecule 1 (VCAM-1), which provides further evidence of a relationship between basal cells and immunocytes. If basal cells have an immunological function, failure of their recruitment into the epididymal epithelium at about the time of puberty may have repercussions for immunological protection of spermatozoa and, ultimately, for fertility in the adult. consistent with the macrophage-like qualities of basal cells of men (Yeung et al., 1994), monkeys (C-H. Yeung and T. G. Cooper, unpublished) and mice (Seiler et al., 1998), is that basal cells originate from the circulation (Nemiloff, 1926), as tissue-fixed macrophages originate from circulating monocytes (Roitt, 1984). Furthermore, human basal cells express CD44 (Terpe et al., 1994), a 'homing receptor' involved in directing lymphocytes to non-lymphoid tissues. Recent evidence has demonstrated a role of the mononuclear phagocyte growth factor, colony stimulating factor 1 (CSF-1), in male reproductive performance, as osteopetrotic transgenic mice with inactivating mutations of this gene $\left(c s f m^{O P} / c_{s f m} O P\right)$ are infertile (Cohen et al., 1996) and effects on the location of peri-epithelial cells in the epididymis have been demonstrated (Cohen et al., 1997).

The aim of the present study was to determine the origin of epididymal basal cells in the mouse model by: (i) investigating the relationship between the change in number of basal cells with the numbers of mitotic figures and peritubular cells during the peri-pubertal period; (ii) labelling putative precursor cell types (monocytes and principal cells) with bromodeoxyuridine before the appearance of basal cells in the epididymis and monitoring the appearance of labelled basal cells; and (iii) immunohistochemical staining of the cytokines possibly involved in cell migration. If basal cells have an extratubular origin, failure of their recruitment into the epididymal epithelium before spermatozoa arrive at 
puberty may have repercussions for immunological protection of spermatozoa and, ultimately, for fertility.

\section{Materials and Methods}

Mice of Balb/c strain were obtained from Charles River (Sulzfeld) and maintained at $22^{\circ} \mathrm{C}$ under $12 \mathrm{~h}$ light: $12 \mathrm{~h}$ dark with access to water and food (Altromin, Lage) ad libitum. All experiments were performed according to the guidelines of the German law on animal experimentation.

\section{Experiment 1: counting of cells and mitoses}

Animals ( $n=4$ of each age) were killed by $\mathrm{CO}_{2}$ asphyxiation and subsequent cervical dislocation on days 14,18 , 22, 26 and 30. Their epididymides were removed, fixed by immersion in Bouin's fluid, washed three times with ethanol, dehydrated in a histomaster (Vieth, Wiesmoor) and embedded in paraffin wax. Sections (3-4 $\mu \mathrm{m}$ thickness) were cut with a Leica SM2000R-microtome (Leica, Nußloch) and stained with periodic acid-Schiff. Transverse sections of 600 tubules in the caput, corpus and cauda epididymides were examined for the number of peritubular cells (closely adherent to the basement membrane) and intra-epithelial basal cells (on the intratubular side of the basement membrane). The number and location (apical or basal) of mitotic figures per tubule cross-section were also evaluated. Valid comparisons between epididymal segments, with their natural differences in tubule size, and between ages, with their timedependent growth, were made by measuring the perimeter of each tubule with a Zeiss Vidas system (KS400; Zeiss, Oberkochem) and the results were expressed per $\mathrm{mm}$ perimeter length.

\section{Experiment 2: labelling of cells with bromodeoxyuridine}

Balb/c mice $(n=12)$ were weighed and injected i.p. on day 20 or 21 of age with bromodeoxyuridine (BrdU: Sigma, Taufkirchen; $1 \%(\mathrm{v} / \mathrm{v})$ solution in sterile $0.9 \%(\mathrm{w} / \mathrm{v}) \mathrm{NaCl}$, $100 \mathrm{mg} \mathrm{kg}^{-1}$ body weight). Animals were killed on days 22 $(n=8)$ and $27(n=4)$. Epididymides were removed, placed in a Petri dish containing PBS, trimmed free of fat and fixed in Bouin's solution overnight. After three washes in $70 \%$ $(\mathrm{v} / \mathrm{v})$ ethanol, the tissues were dehydrated and embedded in paraffin wax. Sections of 3-4 $\mu \mathrm{m}$ thickness were cut and prepared for immunohistochemistry. Sections were deparaffinized, rehydrated through a graded series of alcohol, washed in running tap water and rinsed in deionized water $\left(\mathrm{dH}_{2} \mathrm{O}\right)$. After blocking with endogenous biotin (Dako, Hamburg; performed exactly as indicated by the manufacturer) and endogenous peroxidase $\left(0.3 \%(\mathrm{v} / \mathrm{v}) \mathrm{H}_{2} \mathrm{O}_{2}\right.$ in PBS for $15 \mathrm{~min}$ ), the sections were washed in $\mathrm{PBS}$ and $\mathrm{dH}_{2} \mathrm{O}$ and incubated in $4 \mathrm{~mol} \mathrm{HCl} \mathrm{I}^{-1}$ for $20 \mathrm{~min}$ at room temperature. Sections were immersed in 0.1 mol disodium tetraborate $\mathrm{I}^{-1}$ for $2 \mathrm{~min}$, washed in PBS and pretreated with $5 \%$ $(\mathrm{v} / \mathrm{v})$ normal goat serum (Dako) in $0.1 \%(\mathrm{w} / \mathrm{v}) \mathrm{BSA}$ in PBS for 20 min to block nonspecific binding. BrdU was located with an anti-BrdU-antibody (Dako; monoclonal mouse
anti-BrdU, diluted $1: 30,1 \mathrm{~h}$ ) and detected by using a combination of two biotinylated anti-mouse IgG antibodies (goat anti-mouse IgG, Fab specific; goat anti-mouse IgG, Fc specific; final dilution 1:150; both from Sigma) in $0.1 \%$ $(\mathrm{w} / \mathrm{v})$ BSA in PBS for $1 \mathrm{~h}$ and peroxidase-conjugated streptavidin (Dako; diluted 1:800) in $0.1 \%(\mathrm{w} / \mathrm{v})$ BSA in PBS for $30 \mathrm{~min}$ afterwards. The negative control consisted of mouse isotype Ig (Dianova, Hamburg; CBL 600 mouse $\operatorname{lgG1}$, diluted 1:20). Sections were rinsed in PBS, incubated with substrate (DAB; Sigma) and counterstained with Meyer's haematoxylin. Tissue was washed in $\mathrm{dH}_{2} \mathrm{O}$ and tap water, dehydrated, immersed in paraclear twice for 5 min each time and mounted with a coverslip (Merckoglas; Merck, Darmstadt).

The numbers of basal, peritubular and principal cells with and without nuclear staining for BrdU were counted in up to 1080 tubule cross-sections using a Zeiss Axioskope microscope with $a \times 63$ objective. For each type of cell, the percentage of labelled cells in each epididymal region of each age was calculated. Basal cells were recognized from their position at the base of the epithelium and inside the basement membrane, and by the slightly asymmetric nucleus aligned circumferentially (see BC in Fig. 1a), whereas principal cell nuclei were more circular in profile (see PC in Fig. 1a).

\section{Experiment 3: detection of cytokines}

Epididymides were removed from animals aged 14, $18,22,26$ and 30 days ( $n=20$, four animals each), snap frozen in liquid nitrogen and stored at $-80^{\circ} \mathrm{C}$. Subsequently, $7 \mu \mathrm{m}$ cryosections were cut using a Leica cryostat (Frigocut 2800E). The sections were prepared for immunohistochemistry and stained with antibodies raised to the cytokines as follows. Sections were first air-dried, fixed in acetone for $3 \mathrm{~min}$ at room temperature and pretreated with $0.08 \%(\mathrm{v} / \mathrm{v}) \mathrm{H}_{2} \mathrm{O}_{2}$ in $1 \%(\mathrm{w} / \mathrm{v}) \mathrm{NaN}_{3}-\mathrm{PBS}$ for $20 \mathrm{~min}$ to inactivate endogenous peroxidase. When biotinconjugated antibodies were used (see below), a biotin blocking step (Dako) was inserted after the fixation step to inhibit non-specific binding to endogenous biotin during the immunohistochemical detection of antigens. The sections were rinsed three times in PBS and immersed in $1 \%$ $(\mathrm{w} / \mathrm{v}) \mathrm{BSA}$ in PBS for $1 \mathrm{~h}$ to minimize nonspecific antibody binding. After washing once in PBS, the sections were incubated with the appropriate primary antibody for at least $1 \mathrm{~h}$ at room temperature in a humid chamber. The following primary antibodies and concentrations were used: rat antimouse CD106 (VCAM-1), $1 \mu \mathrm{g} \mathrm{ml}^{-1}$ (Southern Biotechnology Associates (SBA), Birmingham, AL); rat anti-mouse CD54 (ICAM-1), $0.02 \mu \mathrm{g} \mathrm{ml}^{-1}$ (SBA); rabbit anti-mouse interleukin $1 \alpha(\mathrm{IL}-1 \alpha)$ and rabbit anti-mouse IL-1 $\beta$, each $4 \mu \mathrm{g} \mathrm{ml} \mathrm{m}^{-1}$ (Endogen, Cambridge, MA); rat anti-mouse granulocytemacrophage colony-stimulating factor (GM-CSF), $20 \mu \mathrm{g}$ $\mathrm{ml}^{-1}$ (Harlan Sera-Lab, Loughborough), biotinylated rat antimouse gamma-interferon (IFN- $\gamma$ ), $4 \mu \mathrm{g} \mathrm{ml}^{-1}$ (BD Pharmingen, Heidelberg). 

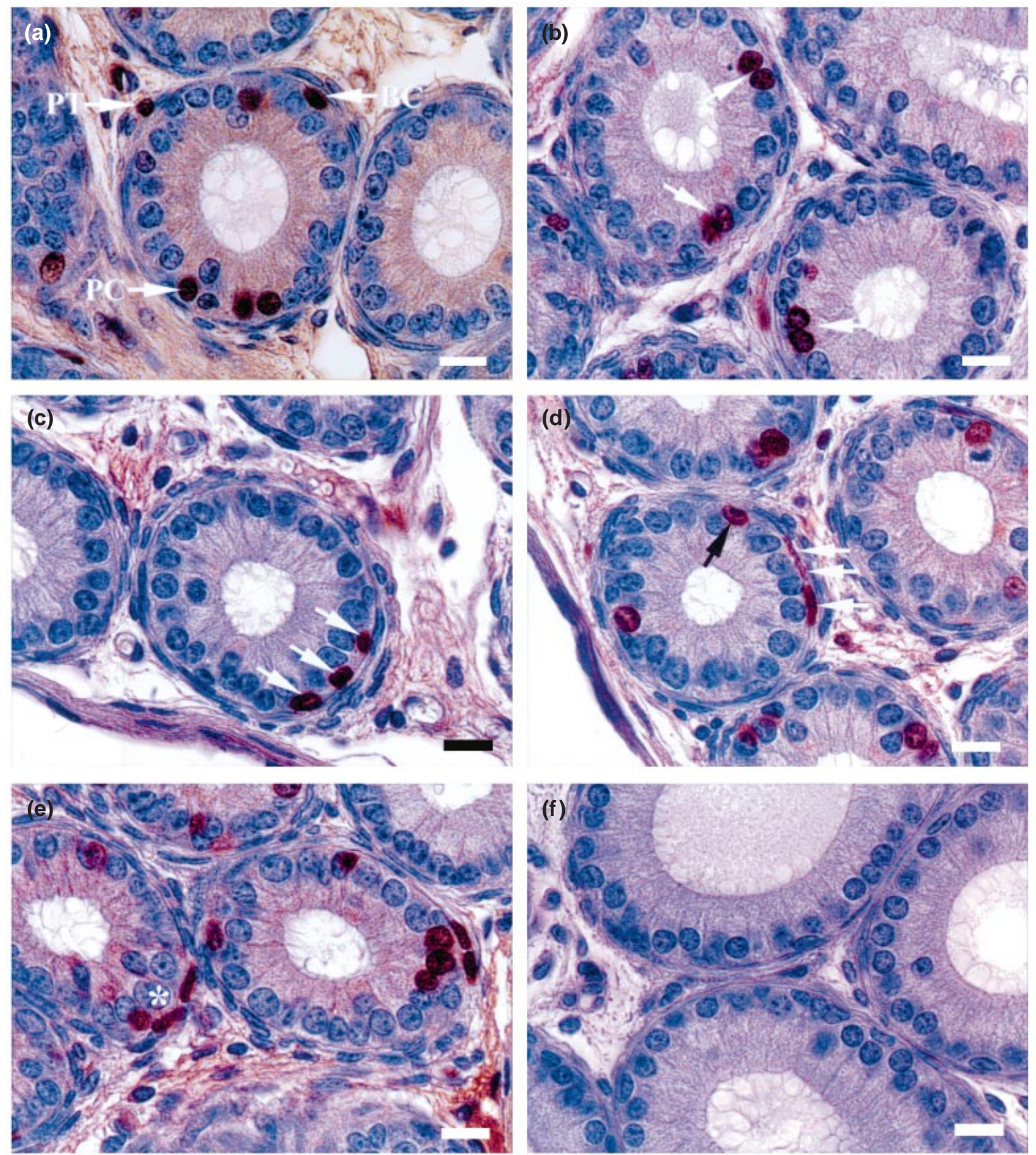

Fig. 1. Photomicrographs of mouse epididymal tissue (caput) removed 1-2 days after bromodeoxyuridine (BrdU) injection and stained with an anti-BrdU antibody. (a) BrdU (brown staining, white arrows) is evident in the nuclei of peritubular cells (PT), principal cells (PC) and basal cells (BC). (b) Labelled principal cell nuclei (arrows) were often observed in side-by-side pairs (arrows) representing daughter cells dividing circumferentially. (c) Labelled basal cell nuclei (arrows) were observed beneath principal cell nuclei that did not contain BrdU. (d) Peritubular cells (white arrows) were often found surrounding tubules in which labelled basal cells (black arrow) were some distance away. (e) This rare section reveals a cell configuration consistent with migration of BrdU-labelled cells across the basement membrane (asterisk). (f) Control sections incubated without primary antibody were not stained. Scale bars represent $10 \mu \mathrm{m}$. 
Sections were washed three times in PBS and cytokines were localized with peroxidase-conjugated secondary antibodies (goat anti-rat IgG or goat anti-rabbit IgG, diluted $1: 100$, for $1 \mathrm{~h} ; \mathrm{SBA})$, whereas in the case of the rat antibody a preincubation with $20 \%(\mathrm{v} / \mathrm{v})$ normal mouse serum (prepared from mice housed in the animal department) and $10 \%(\mathrm{v} / \mathrm{v})$ normal goat serum (Dako), and in the case of the rabbit antibody an incubation with $10 \%(\mathrm{v} / \mathrm{v})$ normal goat serum, for $1 \mathrm{~h}$ was necessary to avoid crossreactivity. When biotinylated primary antibodies were used, the streptavidinhorseradish peroxidase system (Dako; diluted 1:800, $1 \mathrm{~h}$ ) was used as the secondary antibody to get stronger signals.

Negative controls consisted of incubation in the absence of primary antibody (BSA) or irrelevant IgG isotype controls (biotinylated rat IgG1 standard from BD Pharmingen; rat $\lg \mathrm{G} 2 \mathrm{a}$ and rat $\lg \mathrm{G} 1$ isotype controls from SBA). As positive controls for ICAM and VCAM, 12-week-old infiltrates of Leishmania-infected mouse pads (a generous gift from P. D. Sunderkötter, Münster) were used. After three washes in PBS, the sections were stained with 3-amino-9-ethylcarbazole (AEC; Sigma) as chromogenic substrate; endogenous peroxidase was blocked by incubation of the sections with $0.3 \%$ $(\mathrm{v} / \mathrm{v})$ periodic acid-Schiff in $\mathrm{dH}_{2} \mathrm{O}$ for 2 min; sections were washed in water and counterstained with Meyer's haematoxylin. Finally, the sections were mounted in aqueous mounting medium (Faramount; Dako). The location of specific antigen was listed as being epithelial, interstitial, peritubular and associated with cells or connective tissue by using a Zeiss microscope and a $\times 63$ objective.

\section{Detection of macrophage migration inhibitory factor (MIF)}

Epididymides from 14-, 18-, 22-, 26- and 30-day-old animals were fixed in Bouin's fluid and embedded in paraffin wax. Thin sections were prepared as described previously. Blocking of endogenous biotin with Dako blocking solution was done in accordance with the manufacturer's instructions and $0.3 \%(\mathrm{v} / \mathrm{v}) \mathrm{H}_{2} \mathrm{O}_{2}$ in PBS for 15 min was used for blocking of endogenous peroxidase. Primary antibodies were pretreated for 20 min with 5\% normal goat serum (Dako) in $0.1 \%(\mathrm{w} / \mathrm{v})$ BSA in PBS to reduce nonspecific binding that could lead to background staining. Sections were incubated with either monoclonal anti-mouse MIF antibody (diluted 1:50) or polyclonal anti-mouse MIF antibody (diluted 1:10; both a generous gift from P. D. Meinhardt, Marburg) in PBS containing $0.05 \%(\mathrm{v} / \mathrm{v})$ Tween-20 overnight. After several washes in PBS, biotinylated goat antimouse IgG (Fab or Fc specific, final concentration 1:150 for $1 \mathrm{~h}$; Sigma) or biotinylated goat anti-rabbit (diluted 1:300; Sigma) was added, followed by peroxidase-conjugated streptavidin (Dako; diluted 1:800, 1 h).

Negative controls were performed by processing sections identically but instead of the primary antibody, BSA or mouse IgG1 (Dianova; diluted 1:20) was used. After the incubation with DAB (Sigma), sections were counterstained and treated as described earlier.

\section{Statistical analysis}

The Kruskal-Wallis one-way ANOVA on ranks was used to assess differences in cell numbers or mitoses per tubule cross-section or per $\mathrm{mm}$ perimeter with age followed by Dunn's method for multiple comparisons. Differences were considered significant at $P<0.05$.

\section{Results}

\section{Experiment 1: cell counting}

Basal cells were absent from the 14-day-old epididymis and appeared on day 18 in the cauda and corpus epididymides, but not until day 22 in the caput epididymidis (Fig. 2). The numbers reached a maximum of about three per tubule in the cauda epididymidis and two in the corpus epididymidis on day 22, and decreased (cauda epididymidis) or reached a plateau (corpus epididymidis) thereafter. In the caput epididymidis, the number of basal cells per tubule cross-section increased on both day 26 and day 30 . In no region did the number of mitotic figures per tubule cross-section alter during this time, as it remained at about one per tubule in all regions. Mitotic figures were observed within the principal cells only, and no basal cell or peritubular cell divisions were observed.

The number of peritubular cells closely adherent to the basement membrane followed the same pattern in all epididymal regions with about 2.5-3.0 per tubule crosssection on days 14 and 18 , and increasing to a maximum of 4-5 per cross-section on day 22 , which was maintained on days 26 and 30 . The similarity in the patterns of increase in numbers of peritubular and basal cells in the cauda and corpus epididymides was reflected in the increased number of basal cells:number of peritubular cells from day 14 to day 22 , when the number of basal cells was about $50 \%$ of the number of peritubular cells (data not shown). In the caput epididymidis, the delayed appearance of basal cells led to a 4 day shift in this increase, which occurred between day 22 and day 30 (Fig. 2).

When the tubule perimeters were taken into account, the number of peritubular cells per $\mathrm{mm}$ tubule perimeter was similar in all epididymal regions (15-20 cells per $\mathrm{mm})$, decreased on day 18, increased on day 22 and decreased to the original values on days 26 and 30 in all regions (Fig. 3). Numbers of basal cells per $\mathrm{mm}$ perimeter were similar (7-10 cells per $\mathrm{mm})$ in the corpus and cauda epididymides on day 22 , but in the caput epididymidis, the number of basal cells was lower and was still increasing at the end of observation on day 30 . The number of mitotic figures per $\mathrm{mm}$ perimeter was similar in all epididymal regions and decreased gradually from day 18 (corpus epididymidis), 22 (caput epididymidis) or 26 (cauda epididymidis).

\section{Experiment 2: bromodeoxyuridine labelling}

On day 22 the number of basal cells per tubule crosssection was 2.4 in the caput epididymidis, 3.4 in the corpus 


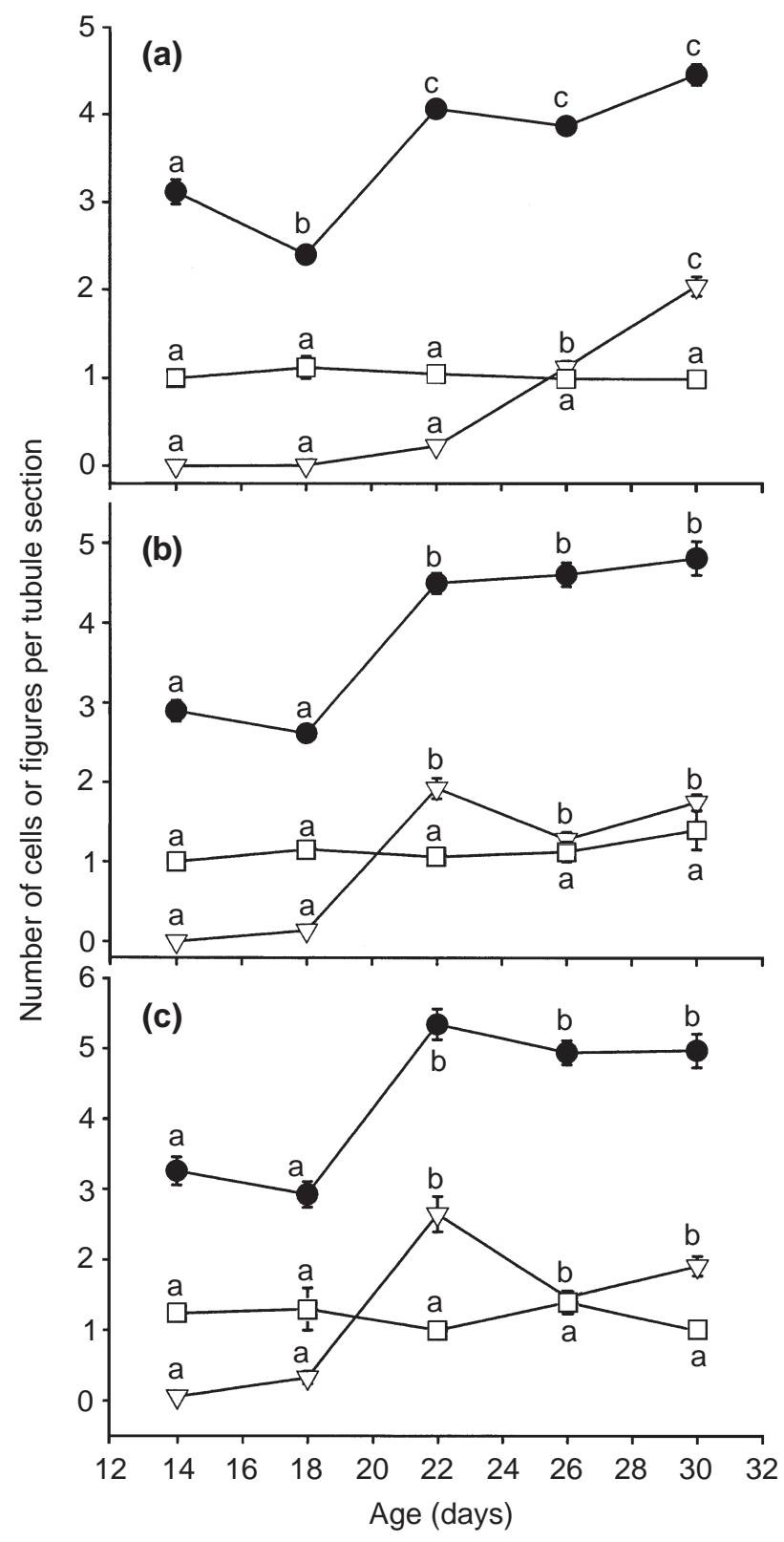

Fig. 2. Numbers of peritubular cells $(\bullet)$, basal cells $(\nabla)$ and mitotic figures $(\square)$, expressed per tubule cross-section, in the mouse epididymis during development (abscissa) in the (a) caput, (b) corpus and (c) cauda epididymides. Values are mean \pm SEM for 1800 tubule cross-sections from 20 mice. abcFor each parameter, values with different superscripts are significantly different $(P<0.05)$.

epididymidis and 3.6 in the cauda epididymidis. A comparison of these data with those obtained from day 27 revealed a significantly higher number of basal cells in the caput (3.2) and corpus (4.4) epididymides on day 27. The number of basal cells in the cauda epididymidis at this time was approximately doubled (6.2) (Fig. 4a). For the principal cells in the epididymis, 22 cells per tubule cross-section were found in the caput epididymidis on day 22 , followed
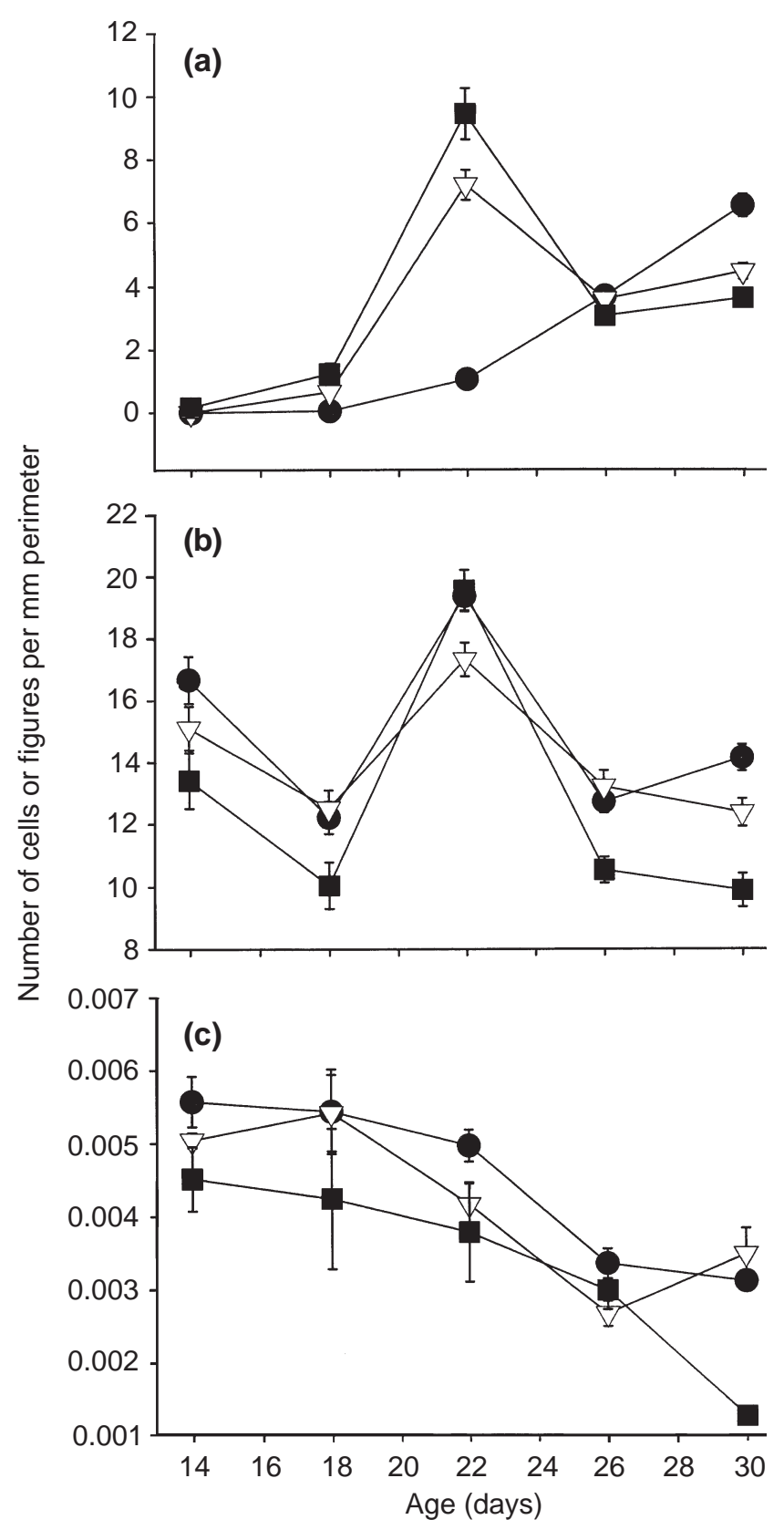

Fig. 3. Numbers of (a) basal cells, (b) peritubular cells and (c) epithelial mitotic figures, expressed per $\mathrm{mm}$ tubule perimeter, in the mouse epididymis during development (abscissa) in the caput (O), corpus $(\nabla)$ and cauda $(\mathbf{\square})$ epididymides. Values are mean \pm SEM for 1800 tubule cross sections from 20 mice.

by 29 cells per tubule cross-section in the corpus epididymidis and 35 cells per tubule cross-section in the cauda epididymidis. The number of this type of cell increased significantly by day 27 in all epididymal regions (caput: 29; corpus: 32 ; and cauda: 39 cells per cross-section). No significant difference in the number of peritubular cells per tubule cross-section was observed on days 22 and 27 . The number of cells per tubule cross-section in the cauda 

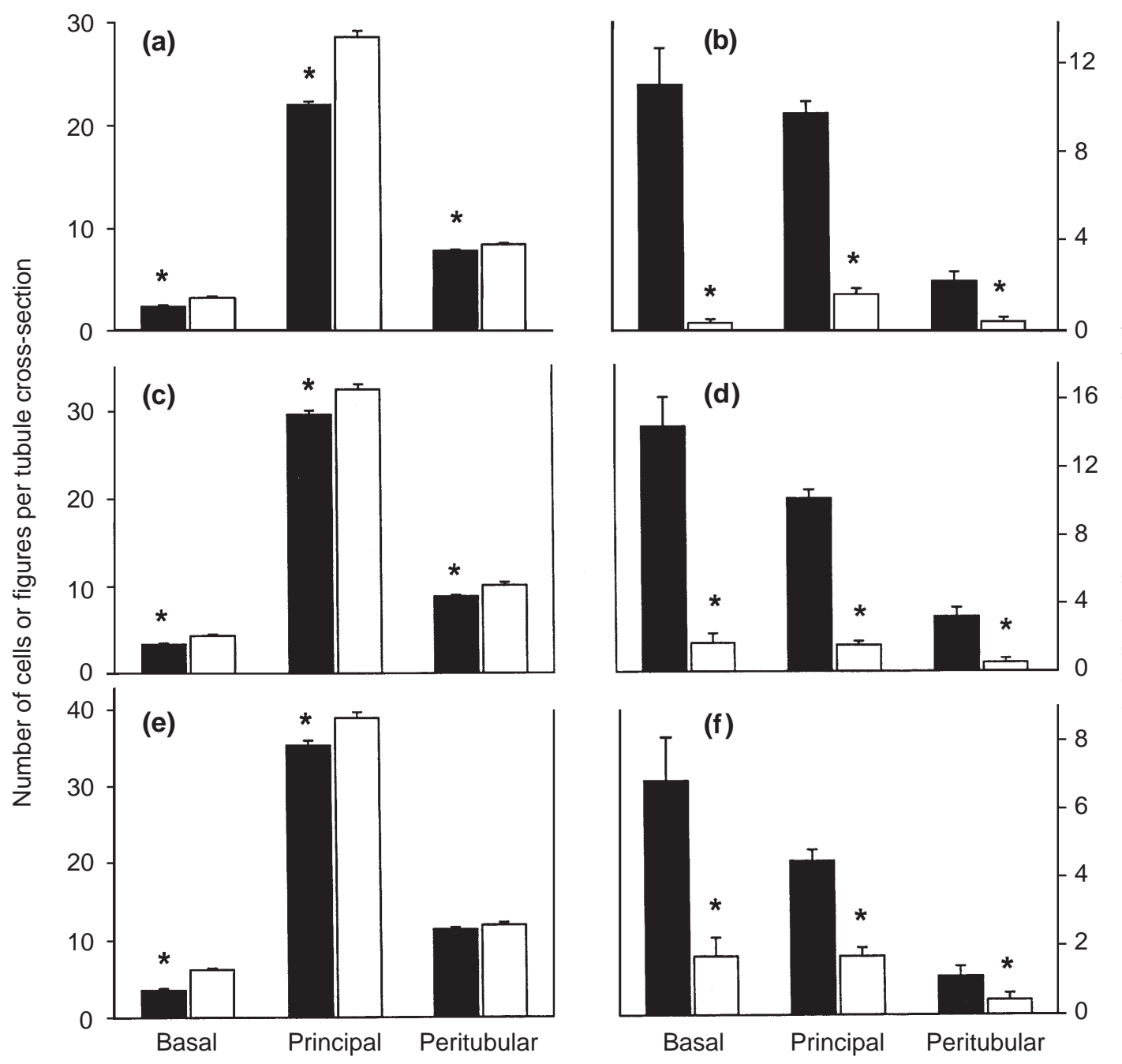

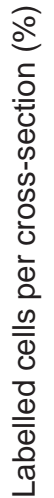

Fig. 4. (a,c,e) Number of cell-specific nuclei per tubule cross-section (ordinate, mean + SEM) in different types (abscissa) in mouse (a) caput, (c) corpus and (e) cauda epididymides on days 22 ( $\square$ ) or 27 ( $\square$ ) after bromodeoxyuridine $(\mathrm{BrdU})$ injection on days 20 or 21. (b,d,f) Number of BrdU-labelled nuclei per tubule expressed as a percentage of the total number of cells of one type (abscissa) per cross-section in the (b) caput, (d) corpus and ( $f$ ) cauda epididymides on days $22(\square)$ or $27(\square)$ after BrdU injection on days 20 or 21 . Values are mean \pm SEM. Asterisks indicate significant differences between the two ages $(P<0.05)$.

epididymidis on both days was nearly identical (11.4 and 11.9) (Fig. 4a), whereas in the caput epididymidis ( 7.7 and 8.3) and corpus epididymidis (8.8 and 10.1), the number of peritubular cells varied slightly but significantly.

The results of the BrdU-labelling, expressed as percentages of the particular total cell population of interest, are shown (Fig. 4b). On day 22, $11.1 \%$ (caput), $14.4 \%$ (corpus) and $6.8 \%$ (cauda) of the basal cells were labelled, but these percentages in all regions had decreased significantly by day 27 to $0.2 \%$ in the caput epididymidis and $2.0 \%$ in the corpus epididymidis and cauda epididymidis. The percentage of labelled principal cells per cross-section was lower than that of the basal cells on day 22 (caput: $9.8 \%$; corpus: $10.2 \%$; cauda: $4.5 \%$ ), with a significant decrease to $2 \%$ of cells in the caput, corpus and cauda epididymides retaining the label on day 27 . The peritubular cells with labelling amounting to $2 \%$ in the caput, $3 \%$ in the corpus and $1 \%$ in the cauda epididymides on day 22 represented the cells with the least labelling per tubule cross-section. The percentage of this type of cell in the three regions of the epididymis decreased significantly to $0.3 \%$ in the caput, $0.5 \%$ in the corpus and $0.4 \%$ in the cauda epididymides on day 27.

Microscopic examination of the epididymal sections collected on day 22 revealed several basal cells with labelled nuclei lying circumferentially, with no labelled principal cell in their vicinity (Fig. 1a,b). No labelled nuclei were situated radially (indicative of a division giving rise to the normal relationship between principal cells and basal cells). Likewise, labelled basal cells were found in tubule regions 

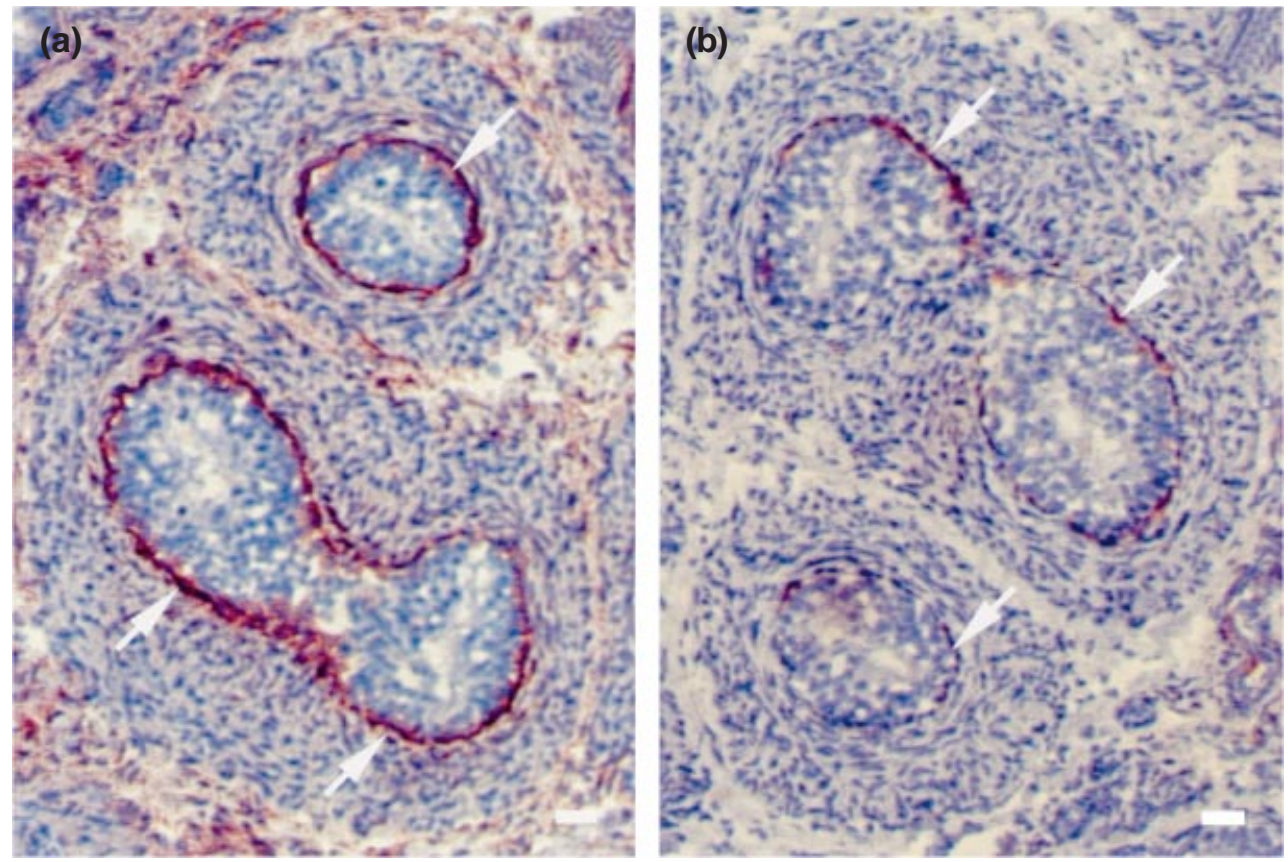

Fig. 5. Photomicrographs of 18-day-old mouse epididymal tissue stained with an antibody to (a) intercellular adhesion molecule 1 (ICAM-1) or (b) vascular cell adhesion molecule 1 (VCAM-1). Note the intense staining of basal cells in the cauda epididymidis (arrows) for both antigens. Scale bars represent $10 \mu \mathrm{m}$.

devoid of principal cell labelling (Fig. 1a,c). Single labelled principal cells were found, but in most cases they appeared in pairs (Fig. 1b). When more than one labelled principal cell nucleus was observed, they were adjacent, indicating that they had divided circumferentially (Fig. 1b,d,e). Labelled peritubular cells in close association with labelled basal cells were never detected (Fig. 1a,d,e), although they were found occasionally beneath labelled principal cells (Fig. 1e). Images indicative of labelled cell translocation across the basement membrane were rare (Fig. 1e).

\section{Experiment 3: detection of cytokines}

Vascular cell adhesion molecule 1 (VCAM-1) was expressed strongly in the cytoplasm of basal cells in the vas deferens on day 14 and persisted until day 22 when labelling decreased (day 26) or was absent. Basal cells in the cauda epididymidis were stained on days 18-26 (Fig. 5a). Basal cells in the corpus and caput epididymides never expressed VCAM-1. Light staining of the connective tissue was present in peritubular tissues.

Like VCAM-1, intercellular adhesion molecule 1 (ICAM-1, CD54) was expressed in basal cell cytoplasm in the vas deferens on days 14-26, in the cauda epididymidis from day 18 to day 26 (Fig. 5b), in the corpus epididymidis from day 22 to day 26, but not in the caput epididymidis, with peritubular staining in all regions throughout (Fig. 5b). The cytokine GM-CSF was localized on days 26 and 30 in the interstitium of the caput, corpus and cauda epididymides but was not associated with basal cells. IL-1 $\alpha$ was only represented in the caudal interstitium on days 26 and 30 at nearly the same intensity. IL-1 $\beta$ was not detected in either the caput or corpus epididymidis of any age. It was observed in the vas and cauda epididymidis associated with peritubular muscle at all times and with stronger expression than IL- $1 \alpha$. Basal cells were negative for both interleukins.

Monoclonal antibodies to MIF reacted positively with all epididymal tissue (epithelium, peritubular muscle in the cauda epididymidis) with more signal from the muscle at older ages. Epithelial staining was greater with the polyclonal antibody. MIF was found in the epithelium of caput and corpus epididymides from day 22 but in the epithelium of cauda epididymidis on days 26 and 30 only. It was also found in the interstitium of caput and corpus epididymides on days 14-22 at a comparable intensity, whereas the interstitium of cauda epididymidis lacked the molecule completely. A peritubular distribution of MIF was monitored in the 14-day-old caput and corpus epididymides as well as in the 22-day-old cauda epididymidis. Connective tissue in the younger cauda epididymidis (days 14-22) was stained less.

Staining with the polyclonal MIF antibody resulted in an equally intense, complete staining of the epithelium in all three epididymal regions at all ages. Only the cauda epididymidis on day 26 had an additional peritubular staining and the 22-, 26- and 30-day-old cauda epididymidis displayed staining associated with connective tissue.

No immunohistochemical staining of IFN- $\gamma$ was detected 
in any region at any time. All negative controls were negative and the positive controls showed positive staining with all the cytokines (not shown).

\section{Discussion}

The present study confirms previous observations that basal cells arise in mouse epididymis after birth and during the first 2 weeks of life (Abou-Haila and Fain-Maurel, 1985; Schantz and Hamilton, 1986), and that they appear first in the cauda epididymidis (Seiler et al., 1998). The results of the present study extend earlier observations by revealing that basal cells were present in the vas deferens on day 14, preceding those in the cauda epididymidis. The numerical discrepancy between the numbers of basal cells per $\mathrm{mm}$ tubule perimeter found in this study and those reported by Seiler et al. (1998) may be related to the use of glutaraldehyde fixation and Epon embedding in the former study, as the superior resolution afforded by semi-thin sections is well known. Nevertheless, the relative differences in numbers of cells between epididymal segments and changes with age observed in the present study are in agreement with the former study. In the present study, other cell types were examined as possible precursors of basal cells.

The increase in the number of peritubular cells (applied closely to the basement membrane) coincident with the increase in number of basal cells in the corpus and cauda epididymides is indicative of a precursor relationship, as they were present before the time of arrival of basal cells in these regions. An increased migration of progenitor basal cells from the interstitium towards the tubule and their subsequent migration across the basement membrane to take up residence in the epithelium could be envisaged, but this is clearly not the case in the caput epididymidis, where a similar temporal migration of peritubular cells occurred that was not accompanied by an ingress of basal cells. Evidence against a peritubular origin of basal cells also comes from the labelling by BrdU, which is incorporated into the DNA of cells in the S-phase at the time of injection. Although the number of BrdU-labelled peritubular cells per tubule cross-section exceeded that of labelled basal cells by a factor of two in all epididymal regions on day 22, when expressed as a percentage of the respective cell populations, there were seven times more labelled basal than peritubular cells in each region, indicating that the latter could not be precursors of basal cells by the process of mitotic division. Thus, the increase in the number of peritubular cells in parallel with the increase in the number of basal cells may be fortuitous and unrelated to the appearance of the latter.

The enumeration of mitotic figures within the epithelium of all epididymal regions showed a fairly constant number per tubule cross-section in all regions and a definite decrease when expressed per $\mathrm{mm}$ tubule perimeter at the time when the number of basal cells was increasing. This lack of increase in the mitotic index in the epithelium at about the time of basal cell appearance is further evidence against basal cells arising from the division of pre-existing columnar epithelial cells. Supporting evidence for this concept was provided from the cells labelled with BrdU, as when two adjacent principal cell nuclei were labelled, they were only ever found in a situation indicative of circumferential division, which is indicative of division into two daughter principal cells. A radial distribution of adjacent labelled cells (consistent with an epithelial progenitor cell dividing to produce a daughter cell beneath it) was never observed.

The higher percentage of basal cells with BrdU-labelled nuclei than either principal or peritubular cells leaves the alternative origin of basal cells from the circulation (Nemiloff, 1926) as a distinct possibility. Images indicative of such translocation across the basal lamina were rare; the process could be rapid, although the factors controlling such cell migration are unclear. Basal cells appear in parallel with the increase in serum androgens reported by Jean-Faucher et al. (1978) but they do not depend on signals emanating from exocrine secretions of the testis (Seiler et al., 1999). In addition to specific recruitment during the inflammatory process, monocytes constitutively leave the bloodstream to enter tissues. Not all become macrophages and 50\% migrate back into the bloodstream (Muller and Randolph, 1999). It is possible that those that do not return to the circulation become basal cells, as suggested from the ultrastructural similarity between basal cells and interstitial macrophages in the human epididymis (Yeung et al., 1994).

It was anticipated that expression of VCAM-1 and ICAM-1 would be upregulated in endothelial cells at about the time of basal cell appearance to direct circulating monocytes into the epididymis, but no such labelling was observed. Surprisingly, the basal cells of the vas deferens, cauda and corpus epididymides expressed both ICAM-1 and VCAM-1, which, like the integrin Mac-1 and late macrophage marker F4/80, are immunologically related antigens expressed by basal cells (Seiler et al., 1999, 2000). Monocytes and macrophages do not normally express ICAM-1 but can do so when stimulated by xeno- or antibiotics (Hubbard, 1999; Rogers et al., 2000). It is too early to state whether the expression of these cytokines by basal cells reflects the immunologically privileged area in which they reside or whether expression is merely required for anchorage of the cells in the epithelium.

The tissue expression of the other cytokines examined was not related to the increasing numbers of basal cells in the epithelium. Peritubular staining of ICAM-1 was consistent at all ages, as was muscular staining of $\mathrm{IL}-1 \beta$ and general staining by MIF. CSF-1 had previously been reported to be necessary for the ordered peritubular alignment of macrophages in mouse epididymis (Cohen et al., 1996), but both GM-CSF and IL-1 $\alpha$ increased their immunohistochemical expression on day 26 in the cauda epididymidis after basal cells were present. No expression in the caput epididymidis was observed before the arrival of basal cells in that region.

The results from these experiments indicate that basal cells may arise from extratubular sources as: (i) there was a 
decrease in the number of mitotic figures in the epithelium as the number of basal cells increased; (ii) division of epithelial cells into daughter cells was circumferential (expected if principal cells were to give rise to daughter principal cells) and not radial (expected if principal cells were to give rise to basal cells); (iii) BrdU-labelled basal cell nuclei were not found in the vicinity of labelled principal cell nuclei and vice versa; (iv) no mitotic figures were observed in the base of the epithelium; (v) the increase in the number of peritubular cells did not parallel the number of basal cells in the caput epididymidis; and (vi) the percentage of labelled basal cell nuclei was higher than that of the other cells, which is indicative of their arrival from a more highly labelled pool, probably of circulating monocytes from the bone marrow.

Thus, immunological infertility at the epididymis may be programmed prepubertally if too few or no basal cells are recruited into the epithelium at this time, reducing the potential for scavenging of sperm autoantigens after puberty and increasing the chances for infertility in the adult.

The authors would like to thank E. Nieschlag, Director of the Institute of Reproductive Medicine for constant support and encouragement, and Margret Kloth and Anne Erpenbeck-Leuer for competent technical assistance. This work was supported by the 'Interdisziplinäres Zentrum für Klinische Forschung (IZKF)', of the Medical Faculty of the University of Münster, Projekt D1 "Chronische Entzündung des Nebenhodens als pathogenetischer Mechanismus männlicher Fertilitätsstörungen".

\section{References}

Abou-Haila A and Fain-Maurel MA (1985) Postnatal differentiation of the enzymatic activity of the mouse epididymis International Journal of Andrology 8 441-458

Clermont Y and Flannery J (1970) Mitotic activity in the epithelium of the epididymis in young and old adult rats Biology of Reproduction 3 283-292

Cohen PE, Chisholm O, Arceci RJ, Stanley ER and Pollard JW (1996) Absence of colony-stimulating factor- 1 in osteopetrotic ( $\mathrm{csfm}^{\mathrm{OP}} / \mathrm{csfm} \mathrm{OP}^{\mathrm{P}}$ ) mice results in male fertility defects Biology of Reproduction $\mathbf{5 5}$ 310-317

Cohen PE, Hardy MP and Pollard JW (1997) Colony-stimulating factor-1 plays a major role in the development of reproductive function in male mice Molecular Endocrinology 11 1636-1650

Hubbard AK (1999) Effects of xenobiotics on macrophage function: evaluation in vitro. Methods 19 8-16

Jean-Faucher C, Berger M, de Turckheim M, Veyssiere G and Jean C (1978) Developmental patterns of plasma and testicular testosterone in mice from birth to adulthood Acta Endocrinologica 89 780-788

Martan J (1969) Epididymal histochemistry and physiology Biology of Reproduction 1 134-154

Muller WA and Randolph GJ (1999) Migration of leukocytes across endothelium and beyond: molecules involved in the transmigration and fate of monocytes Journal of Leukocyte Biology 66 697-704

Nemiloff A (1926) Histo-physiologische Untersuchungen über dne Nebenhoden Zeitschrift für Anatomie und Entwicklungsgeschichte 79 1-43

Rogers PD, Stiles JK, Chapman SW and Cleary JD (2000) Amphotericin induces expression of genes encoding chemokines and cell adhesion molecules in the human monocytic cell line THP-1 Journal of Infectious Diseases 182 1280-1283

Roitt I (1984) Essential Immunology pp 182-183. Blackwell Scientific, Oxford

Schantz IC and Hamilton DW (1976) Epithelial differentiation in the epididymis of the postnantal mouse Anatomical Record 184 522-523

Seiler P, Wenzel I, Wagenfeld A, Yeung C-H, Nieschlag E and Cooper TG (1998) The appearance of basal cells in the developing murine epididymis and their temporal expression of macrophage antigens International Journal of Andrology 21 217-226

Seiler P, Cooper TG, Yeung CH and Nieschlag E (1999) Regional variation in macrophage antigen expression by murine epididymal basal cells and their regulation by testicular factors Journal of Andrology 20 738-746

Seiler P, Cooper TG and Nieschlag E (2000) Sperm number and condition affect the number of basal cells and their expression of macrophage antigen in the murine epididymis International Journal of Andrology 23 65-76

Sujarit S and Jones RC (1991) $\left[{ }^{3} \mathrm{H}\right]$ Thymidine uptake by the epididymis, seminal vesicles and prostate gland during postnatal development of the rat Reproduction, Fertility and Development 3 313-319

Sun EL and Flickinger CJ (1982) Proliferative activity in the rat epididymis during postnatal development Anatomical Record 203 273-284

Terpe H-J, Stark H, Prehm P and Guenthert U (1994) CD44 variant isoforms are preferentially expressed in basal epithelial of non-malignant human fetal and adult tissues Histochemistry 101 79-89

Yeung $\mathrm{CH}$, Nashan D, Cooper TG, Sorg C, Oberpenning F, Schulze $\mathbf{H}$ and Nieschlag E (1994) Basal cells of the human epididymis - antigenic and ultrastructural similarities to tissue-fixed macrophages Biology of Reproduction 50 917-926

Received 12 July 2001.

First decision 8 November 2001.

Accepted 13 December 2001. 